\title{
Biases in pigeon working memory
}

\author{
J. GREGOR FETTERMAN \\ Indiana University-Purdue University, Indianapolis, Indiana
}

\begin{abstract}
The psychophysics and short-term retention of pigeons' responses to rate of stimulus change were assessed in two experiments, using a symbolic delayed matching-to-sample procedure. In Experiment 1, the birds discriminated between steady and flickering lights. Psychophysical assessments of the discrimination suggested a mix of analogical and categorical strategies. Retention tests revealed a consistent bias to respond to the choice associated with the flickering sample. In Experiment 2, the birds discriminated between different rates of stimulus change (slow vs. fast). Retention tests indicated a bias to respond to the alternative associated with the slow sample. Transfer tests to new stimulus values revealed that the birds processed the stimuli in an absolute, rather than a relational fashion. These findings are related to recent work on biased forgetting in animal working memory experiments.
\end{abstract}

Since a seminal paper by Blough (1959), the delayedmatching-to-sample (DMTS) procedure has become a standard technique for studying working memory processes in nonhuman animals (e.g., Kendrick, Rilling, \& Denny, 1986; Roberts \& Grant, 1976). In a DMTS procedure, subjects receive a series of trials that begin with the presentation of a to-be-remembered sample event (e.g., a red light); following sample presentation, subjects experience a retention interval that varies across trials (sometimes, across sessions) from $0 \mathrm{sec}$ (immediate test) to some maximum value, typically in the range of 10-20 sec. At the end of the retention interval, two comparison stimuli are presented (e.g., red and green lights); a response to one comparison stimulus is correct after one of the samples, whereas a response to the other comparison stimulus is correct after the other sample. Correct comparison responses lead to reinforcement, whereas incorrect responses are not reinforced, simply initiating an intertrial interval (ITI).

Accuracy of comparison choice decreases with increases in the retention interval (see, e.g., Roberts \& Grant, 1976), an outcome typically interpreted as reflecting a decline in the strength of a working memory code for the immediately prior sample (but see Zentall, 1997, for an alternative interpretation). Normally (e.g., Roberts \& Grant, 1976; Urcuioli \& Zentall, 1986), increases in a retention interval produce overlapping and parallel retention functions for each of the samples. More precisely, the slopes and intercepts of individual sample retention functions are comparable. In other DMTS experiments, however, one sample is retained better than another (i.e., the retention function for one sample is steeper than that for the other). This memory differential-

This research was supported by NSF Grant BNS 9407527. Correspondence concerning this article should be addressed to J. G. Fetterman, Department of Psychology, Indiana University-Purdue University, 402 N. Blackford Street, Indianapolis, IN 46202 (e-mail: gfetter@ iupui.edu). biased forgetting-is of some general interest, because it may provide a window on coding processes in animal working memory (see, e.g., Grant, Spetch, \& Kelly, 1997).

Spetch and Wilkie (1983) provided the prototype demonstration of this phenomenon. In their experiment, pigeons were trained to discriminate the duration of a short- from a long-duration light by reinforcing one choice after the short-duration signal and another after the longer signal. When comparison stimuli were delayed from signal offset, accuracy on short-signal trials remained roughly constant with increases in the retention interval (slope close to zero), whereas accuracy following long signals showed a sharp decline (negative slope) to levels below chance performance. Spetch and Wilkie called this working memory bias the choose-short effect and offered a hypothesis termed subjective shortening as an explanation for the result (see Grant et al., 1997, and Spetch \& Rusak, 1992, for further details). Similar analogical memory biases also occur with delayed discriminations along the numerosity dimension, in which the number of sample responses (Fetterman \& MacEwen, 1989) or the number of light flashes (Roberts, Macuda, \& Brodbeck, 1995) serve as the discriminative cue. When pigeons receive delayed testing for numerosity samples, they tend to report that the prior sample was the smaller of two numerosities, a choose-small effect.

Memory biases have also been observed with presence/absence discriminations. In these tasks, the subject (typically, a pigeon) is asked to choose between two comparison stimuli on the basis of whether a trial began with or without the occurrence of some event, such as the presentation or the omission of a keylight sample. One choice is rewarded on trials initiated by the presentation of the sample, and another on trials in which the sample is not presented. Although not commonly described as such, these are psychophysical detection tasks (Macmillan \& Creelman, 1991). When delays are inserted between trial onset and choice, pigeons tend to select the no-sample comparison, irrespective of the occurrence or nonoccur- 
rence of the sample event (see, e.g., Grant, 1991; Sherburne \& Zentall, 1993). This choose-nothing bias may seem peculiar, because, as Zentall (1997) observes, the presence of a sample seems more salient than its absence and, hence, should be more readily retained. But, as Grant (1991) and Sherburne and Zentall point out, pigeons may adopt a strategy of remembering in which only the presence sample is coded at trial onset, responding to the absent comparison by default. Such a single-code default strategy would support the observed bias when presence/absence samples must be discriminated after a retention interval.

On the face of it, memory biases in timing/counting and presence/absence discriminations might seem to result from different memory processes, and indeed, different explanations have been offered for choose-short (e.g., Spetch \& Rusak, 1992) and choose-nothing (e.g. Sherburne \& Zentall, 1993) effects. Wixted and Dougherty (1996; see also Wixted, 1993) argue, however, that the various instances of biased memory can be explained by a common set of principles based on the relative saliences of sample events. The basic idea is that discriminations in biased-forgetting experiments involve stimuli that differ in salience. When retention intervals are placed between samples and choices, the effect is to reduce the remembered salience of the samples in such a way that the memory of the more salient sample increasingly comes to resemble that of the less salient sample, and memory for the less salient sample disappears altogether (what Wixted and Dougherty refer to as "the mental state of nothingness"). It is further assumed that the samples can be represented on a continuum of salience and that the less salient event is more similar to nothing (no event) than is the more salient event. These assumptions are sufficient to produce different varieties of biased forgetting, which, according to Wixted and Dougherty, reflect tendencies to select the comparison associated with the least salient sample. The notion of salience as a mediator of discriminating and remembering is appealing by virtue of its simplicity in representing both immediate and delayed discriminations along a single dimension.

The experiments in this paper were inspired by biasedforgetting phenomena and, in particular, by the no-sample bias observed with presence/absence discriminations. Presence/absence discriminations involve a contrast between some sample event (e.g., light or food) and the absence of that event (e.g., no light or no food), but such a discrimination may be operationalized in other ways. For instance, a different kind of presence/absence discrimination could involve a dynamic stimulus discriminated from a static stimulus or a distinction between the presence and the absence of stimulus change. A familiar example is the difference between a movie and a still photograph.

In the present experiments, pigeons learned to discriminate a static from a changing stimulus (Experiment 1) and between different rates of change (Experiment 2). Once the discriminations were acquired, retention tests were given to test working memory for the samples. Both experiments included psychophysical assessments of the discrimination as a way of assessing dimensional stimulus control. The main question was whether the pigeons would exhibit biased forgetting of the stimuli.

\section{EXPERIMENT 1}

In the first experiment, pigeons learned a discrimination between steady and flickering lights, which could be construed as the presence versus the absence of stimulus change. Once the task was learned, the birds received delayed presentations of the comparison stimuli. If pigeons respond to the steady and the flickering samples as they do to, for example, light and no-light samples, the birds should show better retention of the steady sample (absence of change) than of the flickering sample (presence of change).

Experiment 1 was very similar to one conducted by Blough (1959), which was referred to at the beginning of this article. In Blough's experiment, pigeons were trained on a nonsymbolic DMTS arrangement in which both the sample and the comparison stimuli consisted of steady and flickering lights. As the focus of the first experiment concerned the possibility of biases in memory for steady and flickering samples, it would seem that Blough's data should settle the question. However, Blough (D. S. Blough, personal communication, June 10, 1997) did not record the data at the level of detail necessary to calculate accuracy scores separately for each sample, the main focus of this experiment.

\section{Method}

\section{Subjects}

The subjects were 5 adult male Silver King pigeons (Columba livia) maintained at approximately $85 \%$ of their free-feeding weights. The pigeons received unlimited access to water and grit in their home cages and (when necessary) supplemental feedings to maintain the animals at their experimental weights. All the birds had prior experimental histories of responding on various schedule-related procedures. None had experience with delayed discrimination tasks. The pigeons were maintained on a 12:12-h light:dark cycle, with light onset at 7:00 a.m.

\section{Apparatus}

The experimental enclosure was a standard BRS-LVE three-key pigeon chamber; the work space measured $32 \mathrm{~cm}$ high $\times 34 \mathrm{~cm}$ wide $\times 34 \mathrm{~cm}$ deep. The keys were accessible through $2-\mathrm{cm}$ circular openings in the work panel on the front wall, with the center of the openings spaced $6.3 \mathrm{~cm}$ apart, $24 \mathrm{~cm}$ above the chamber floor. A force of approximately $0.15 \mathrm{~N}$ was required to operate each of the keys. Standard 28-V lights could illuminate the left and right keys with red, blue, or amber light. Center key illumination was provided by red, green, and amber light-emitting diodes. The feeder opening was located directly below the center response key and measured $5 \mathrm{~cm}$ on all dimensions; the bottom of the feeder opening was $10 \mathrm{~cm}$ above the chamber floor. White noise served to mask extraneous sounds; additional masking and ventilation were provided by an exhaust fan attached to the chamber wall. Experimental events were 
scheduled and recorded by an IBM PC and interface located in an adjacent room.

\section{Procedure}

All the birds had prior experimental training and thus were placed directly on the task described below. Trials began with the illumination of the center key with green light. A peck to the lighted key changed the key color to amber and initiated the sample, which lasted $4 \mathrm{sec}$. On some trials, the sample consisted of a steady amber light, and on other trials, the sample consisted of a flickering $(4.25-\mathrm{Hz})$ amber light. The flicker rate is well below the pigeon's fusion threshold (see, e.g., Powell \& Smith, 1968). The flickering stimulus consisted of equal light-on and light-off periods. Trials were equally divided between steady and flickering samples, and the sequencing of sample types was pseudorandom, under the constraint that each block of 12 trials should contain equal numbers of each sample. After a sample was presented, the center key was darkened, and the left and right keys were illuminated with red and blue lights; the position of the red and blue lights alternated randomly across trials. A response to one key color was correct after steady samples, and a response to the alternate key color was correct after flickering samples (symbolic matching arrangement); the mapping of sample type to comparison key color was counterbalanced across birds. In the initial stage of training, every correct response was reinforced with a 3 -sec access to mixed grain; food access was followed by a 10-sec ITI, during which all the lights were off and responses had no scheduled consequences. Incorrect responses initiated the ITI directly. Sessions ended after 120 trials.

The acquisition criterion was $80 \%$ correct responses for 5 consecutive sessions. Three pigeons met the criterion after fewer than 15 sessions of training, a 4th required 32 sessions, and 1 pigeon (P38) required 84 sessions to achieve the criterion. Ten additional sessions of training were given after the criterion was met.

Psychophysical probe test. Once the basic discrimination was established, the task was modified so that $50 \%$, rather than $100 \%$, of correct choices yielded reinforcement. Correct choices on nonreinforced trials simply produced the ITI, as did all incorrect choices. When the pigeons adapted to the changes in reinforcement, probe trials were substituted for nonreinforced trials. Probe trials contained sample stimuli that flickered at a rate slower than the trained flicker value, and probe trials made up $50 \%$ of the trials in each test session. Four probe stimuli were used $(1.375,1.625,2.12$, and $2.875 \mathrm{~Hz}$ ), and each probe stimulus occurred 15 times during each test session. Choices on probe trials were recorded but never reinforced. Probe sessions alternated with training sessions, providing $50 \%$ reinforcement for choices, and a total of five probe sessions were conducted.

Delay Test I. Once psychophysical testing was completed, all the pigeons were returned to the original testing conditions that provided reinforcement for each correct choice and included two sample types, steady and flickering lights. After 10 sessions of such reminder training, delay testing was initiated. During delay test sessions, $50 \%$ of the trials included a delay between the termination of the sample and presentation of the comparison stimuli; all lights were off during delay intervals. Delays of 2,5 , and $12 \mathrm{sec}$ were used, and each delay and sample type occurred equally often within each session (i.e., 10 presentations of each sample type at each delay). The remaining trials involved an immediate $(0-\mathrm{sec})$ test of sample discrimination. Immediate and delayed test trials were randomly intermixed within sessions, and the first phase of delay testing lasted 5 sessions.

Multiple sample durations. Variable sample durations were introduced following the first delay test with a constant (4-sec) sample duration. Samples could last 2, 4, or $6 \mathrm{sec}$, and each sample duration occurred equally often within each session. Comparison stimuli were presented immediately after the sample was terminated ( 0 -sec delay), and every correct choice was reinforced. This phase of training last 20 sessions.

Delay Test II. This phase of testing was identical to Delay Test I, except that sample duration varied across trials. Each sample type (steady vs. flickering) and sample duration $(2,4$, and $6 \mathrm{sec})$ occurred equally often at each delay. The second phase of delay testing lasted 10 sessions.

A .05 level of significance was used for all statistical comparisons. All post hoc comparisons were carried out according to Tukey's procedures for comparisons of unconfounded means.

\section{Results}

The discrimination was not difficult for the birds to learn, and all the pigeons attained high levels of accuracy. Figure 1 shows the psychophysical data as the probabil-

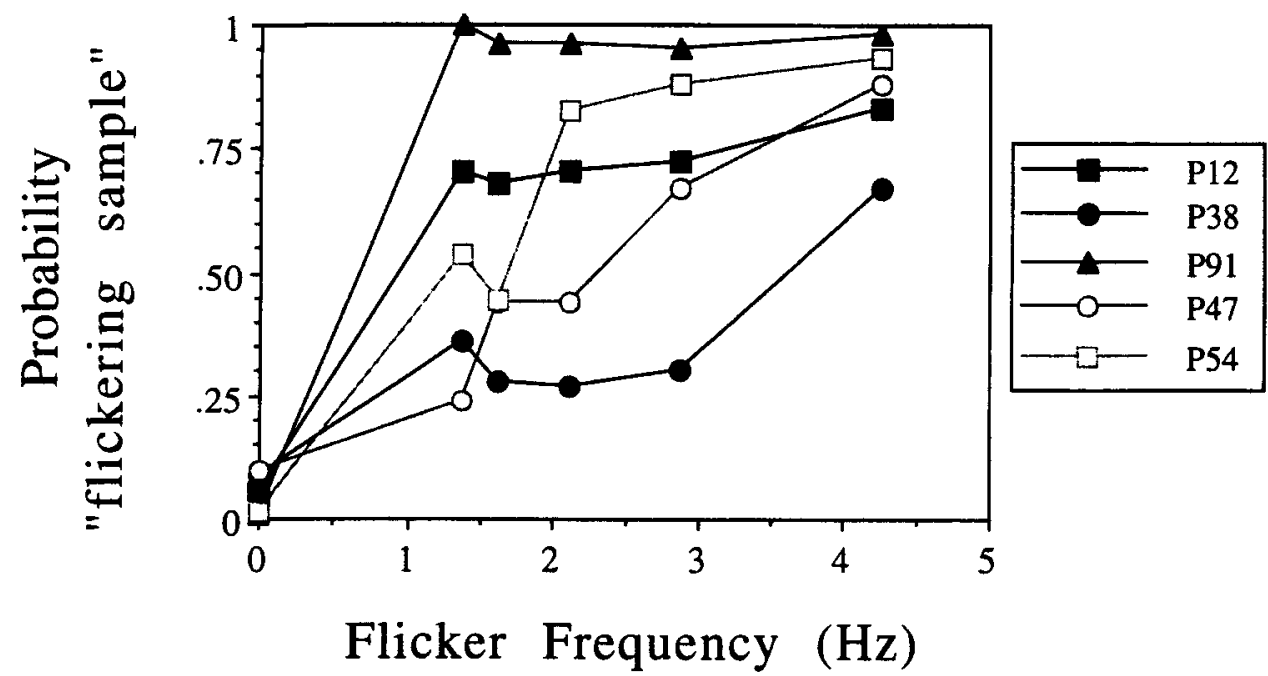

Figure 1. Probability of responding to the alternative associated with the flickering sample as a function of the flicker frequency of training and test stimuli. Probabilities were calculated by dividing the number of choices of the alternative with the flickering sample at each stimulus value by the total number of occurrences of each value. Data are shown for individual pigeons, represented by different symbols. 
ity of choosing the flickering alternative as a function of flicker frequency; the zero value on the abscissa identifies the steady sample. The filled and unfilled symbols identify subgroups of pigeons that demonstrated different choice patterns in relation to changes in flicker frequency. Some of the curves (filled symbols) can be characterized as step functions, indicating a discrete classification of steady and flickering samples (P12, P38, and P91). Pigeons 12 and 91 tended to identify all the flickering samples, irrespective of stimulus frequency, as being equivalent to the trained flicker value $(4.25 \mathrm{~Hz})$. Pigeon 38 classified intermediate flicker rates similarly (function is flat over the range of test stimuli) but showed a clear dis-
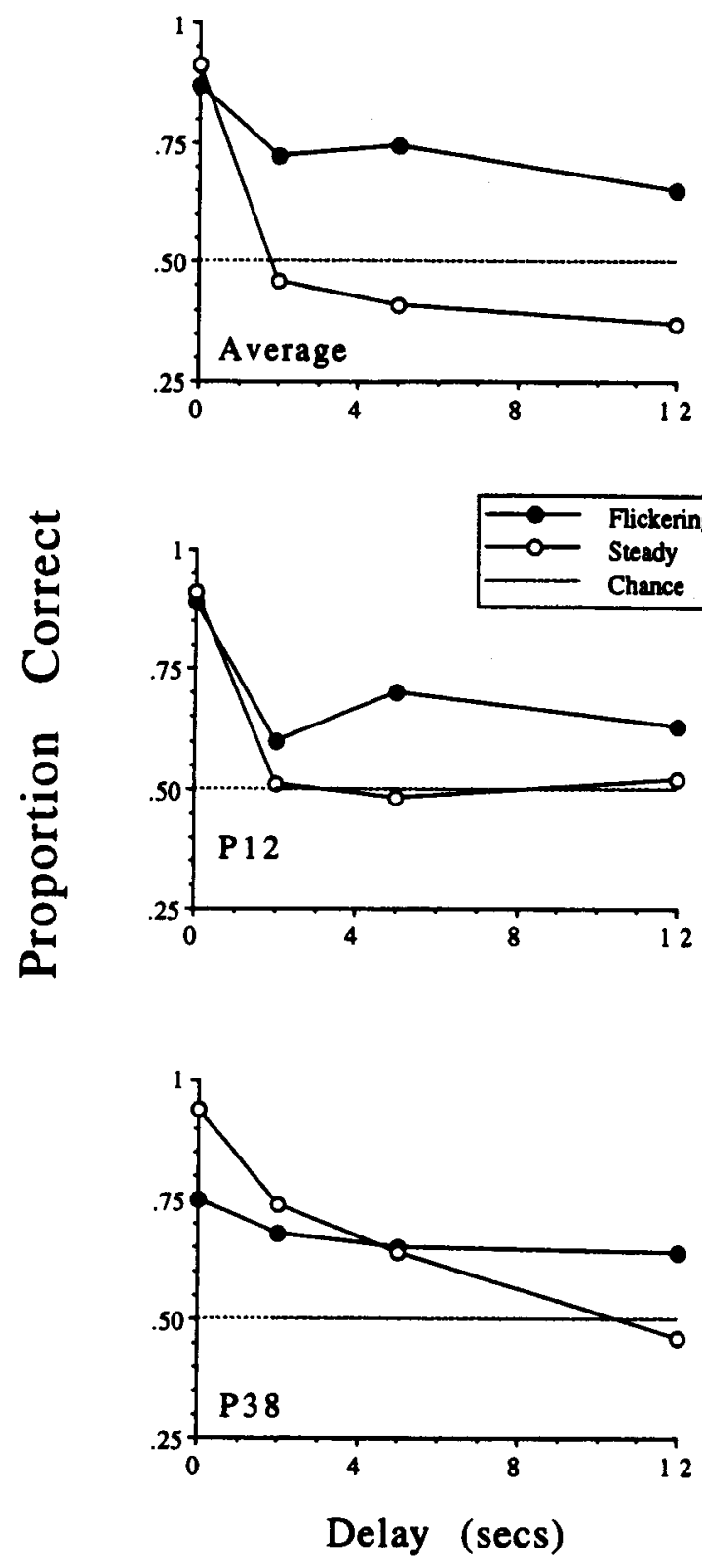

crimination between the trained values ( 0 vs. $4.25 \mathrm{~Hz}$ ), a different kind of categorical response pattern. Pigeons 47 and 54 (unfilled symbols) showed a more graded pattern of choice, reflecting sensitivity to the dimension of stimulus frequency, rather than a categorical classification of the samples.

Figure 2 shows the proportion of correct choices as a function of delay, with separate functions for each sample type. There were clear differences in retention according to sample type. When the sample was a flickering light, accuracy remained above chance, even at the longest $(12-\mathrm{sec})$ delay. When, however, the subjects received delayed testing after a steady sample, accuracy
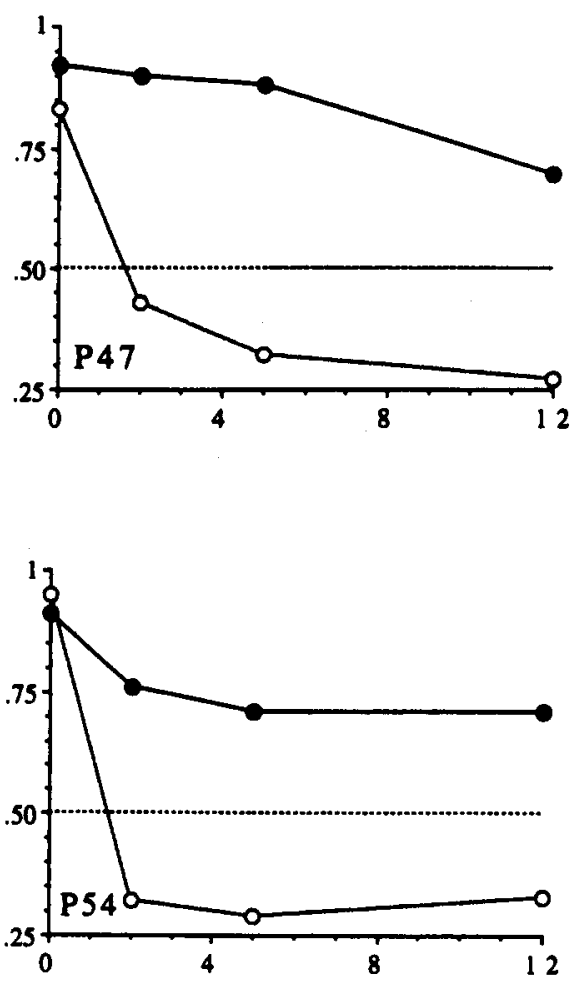

Figure 2. Proportion of correct choices as a function of the delay between the sample stimulus and the comparison stimuli. The data are shown separately for trials initiated by a flickering sample and those initiated by a steady sample. 
was at or below chance levels for 4 of 5 birds after just a 2 -sec delay between the sample and the comparison choice. A two-way analysis of variance (ANOVA), with sample type and delay as the factors, showed that the main effects of sample $[F(1,4)=8.36]$ and delay $[F(3,12)=$ $40.69]$ were significant, as was their interaction $[F(3,12)=$ 18.55]. Sample-delay interactions are the signature of biased forgetting, and post hoc comparisons showed that accuracy following flickering samples was significantly higher than that after steady samples at all delays, except the 0 -sec delay.

After training with a fixed (4-sec) sample duration, the subjects received a regimen of variable sample durations $(2,4$, and $6 \mathrm{sec})$ intermixed within sessions. Figure 3 shows the results from delay tests with mixed sample durations, where accuracy scores are collapsed over sample type. Accuracy declined with increasing delay, and the extent of the decline was related to sample duration. Shorter sample durations produced lower accuracy than did longer sample durations. A two-way ANOVA demonstrated that the main effects of delay $[F(3,12)=30.16]$ and sample duration $[F(2,8)=7.78]$ were significant. The interaction of these variables was not significant.

Figure 4 shows retention functions for each sample duration according to whether the sample was a steady (unfilled symbols) or flickering (filled symbols) light. As in Figure 2, the functions are asymmetrical, but sample duration does not appear to have influenced the degree of asymmetry. Such a result would be reflected in a significant three-way interaction between sample type, sample duration, and delay. A three-way ANOVA revealed significant main effects of sample type $[F(1,4)=$
18.35], sample duration $[F(2,8)=6.52]$, and delay $[F(3,12)=28.48]$, but no significant interactions.

\section{Discussion}

The present experiment produced a biased retention pattern (Figures 2 and 4 ) that could be interpreted in terms of some specific memory code, but the psychophysical data (Figure 1) provide no support for the conclusion that all the pigeons responded to (coded) the samples in the same way. Those data indicated that some birds showed a step-wise function in response to changes in flicker rate, suggesting that they categorized the samples according to the presence or absence of flicker. Other birds showed graded functions, suggesting that they responded to the dimension of flicker rate in an analogical fashion. Despite these apparent differences in sample stimulus control under immediate test conditions, all the birds displayed the same sample bias effect under delayed testing conditions - a preference for the alternative associated with the flickering sample. The bias appeared during the first session of retention testing, making it unlikely that some of the birds adopted a different method of stimulus coding after sample-choice delays were introduced.

Discriminations between flickering and steady lights could be construed as involving a presence/absence discrimination, but this interpretation is not supported by the results of delay tests (Figure 2) that revealed a bias to remember the presence of stimulus change (flickering light) but to forget its absence (steady light). Neither are the results compatible with a theory of biased forgetting based on event salience (Wixted \& Dougherty, 1996), which stipulates that a more salient stimulus is forgotten

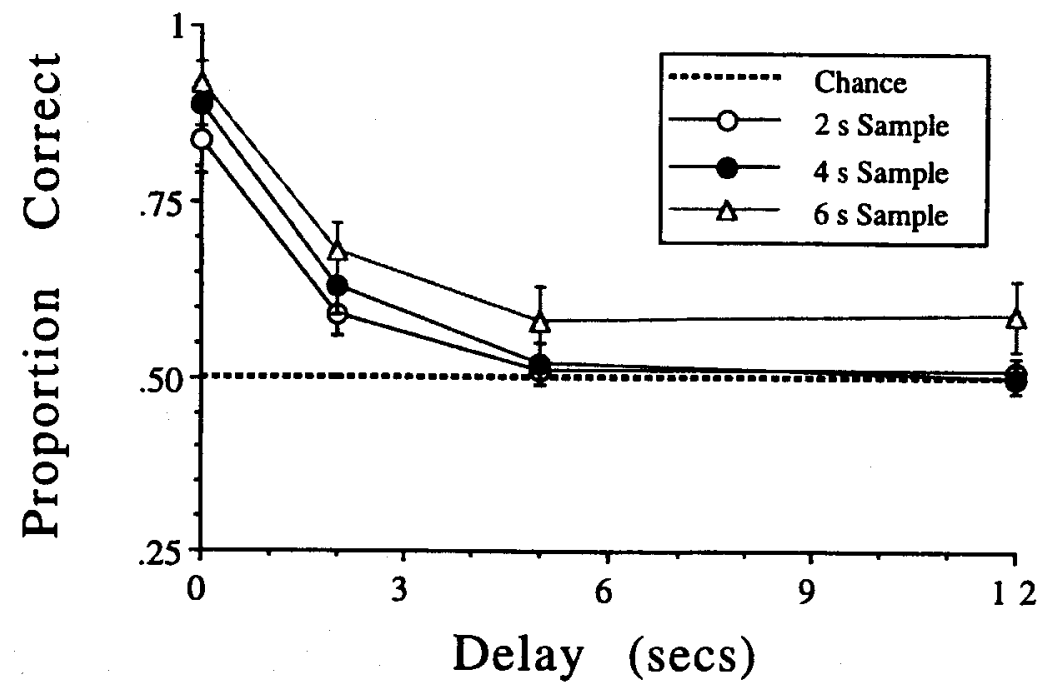

Figure 3. Proportion of correct choices as a function of the delay between the sample stimulus and the comparison stimuli, with sample duration as the parameter. The vertical bars signify \pm 1 standard error of the means. 

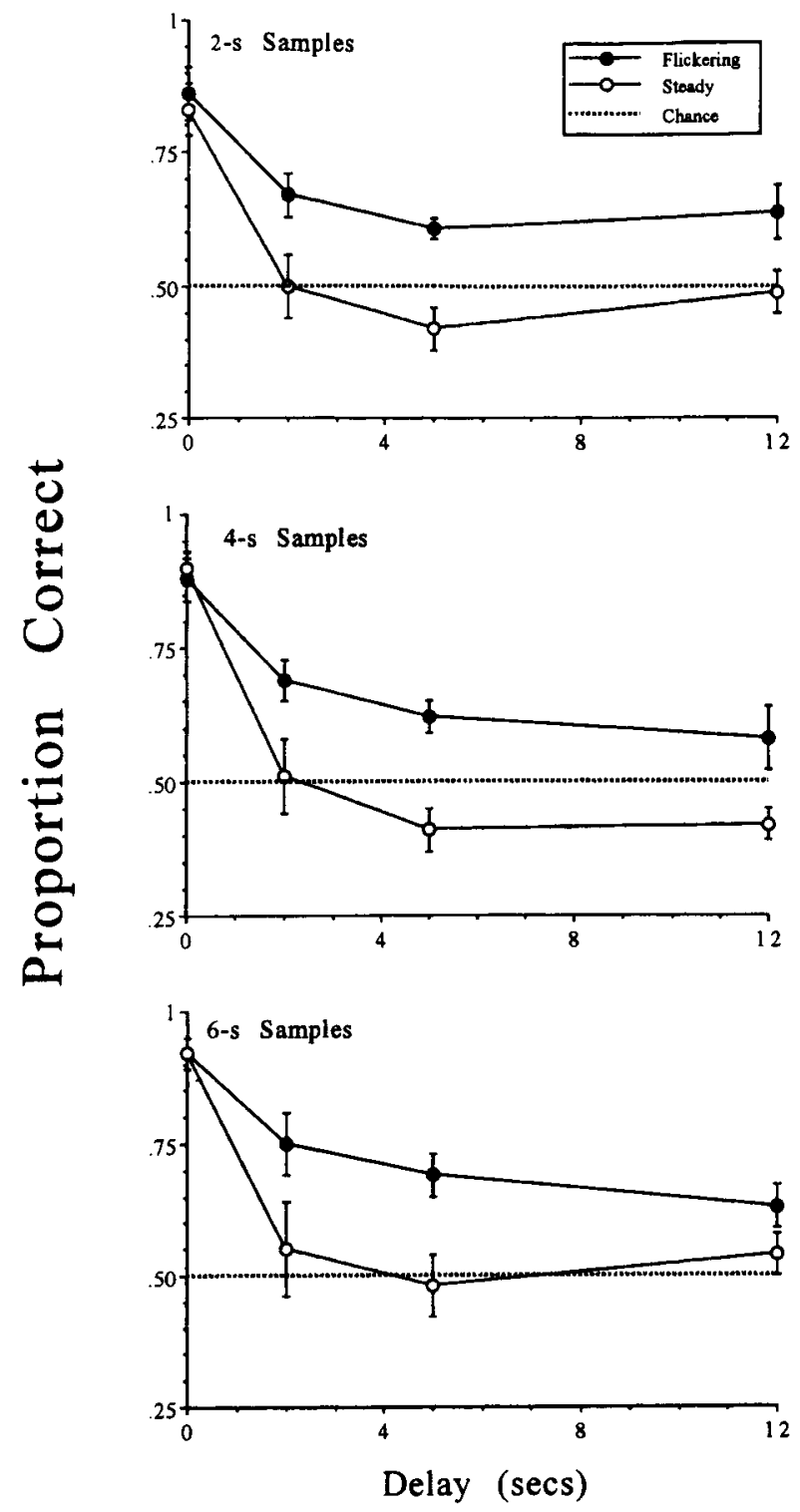

Figure 4. Proportion of correct choices as a function of the delay between the sample stimulus and the comparison stimuli, with sample duration as the parameter. The data are shown separately for trials initiated by a flickering sample and those initiated by a steady sample. The vertical bars signify \pm 1 standard error of the means.

more quickly than a less salient stimulus. The present results demonstrate an asymmetrical retention pattern favoring the flickering stimulus, which, a priori, would seem to be more salient.

The Wixted/Dougherty (1996) model provides a parsimonious account of results from seemingly diverse working memory tasks, but it does not address the issue of determining which of two sample events is more salient, even though event salience is fundamental to the model. Relative event salience sometimes seems obvious, when, for example, a longer event must be discriminated from a shorter event. But we must be cautious about making a priori judgments about stimulus salience, because this is a behavioral question. If a theory of biased forgetting on the basis of event salience is to be taken seriously, it must specify procedures for assessing event salience in advance of retention tests, which may or may not reveal differential rates of forgetting. Otherwise, the model cannot escape the criticism of circularity.

An alternative view of the discrimination is that the birds treated the stimuli as endpoints on a continuum of flicker frequency, although the psychophysical data suggest that not all the birds used this rule. Honig and Spetch (1988) provide data relevant to this interpretation. In their experiment, pigeons discriminated between the rate of alternation of red and green hues. On some (fast) trials, the stimuli alternated every $0.5 \mathrm{sec}$ (one complete cycle each second); on other (slow) trials, the stimuli alternated every $2 \mathrm{sec}$ (one complete cycle every $4 \mathrm{sec}$ ). The number of cycles per trial was varied, in order to avoid a confounding between sample duration and cycle rate. Different comparison stimuli were correct after slow and fast samples, and when delays were placed between the samples and the comparisons, the birds demonstrated a bias to select the fast comparison. The results of Experiment 1 may be viewed as corroborative of the findings of Honig and Spetch, assuming that the birds treated the steady sample as slow and the flickering sample as fast.

Honig and Spetch (1988) interpreted this result in terms of a subjective shortening process involving the component durations, a process that has been used to explain the choose-short effect in memory for single stimulus durations (e.g., Spetch \& Wilkie, 1983). By their interpretation, then, choices were determined by the remembered values of the components of the cyclic samples, and not by the higher order dimension of rate of change.

However, the bias observed in this experiment may not reflect a difference in memory but, rather, the fact that all the pigeons used a single-code default rule-if the sample was steady, respond to comparison $x$; otherwise, respond to comparison $y$. As memory for the steady sample fades, a pigeon would increasingly make the default response, resulting in high accuracy on flickering sample trials and below chance accuracy on steady sample trials. This scenario would explain the memory biases but would not explain why all the pigeons would adopt the rule in the first place. Moreover, the psychophysical data suggest that not all the pigeons used the same rule.

Sample duration affected the overall accuracy of delayed discrimination but did not influence choice bias. Longer sample durations produced better retention of the samples, but sample duration did not interact with sample type and delay. Sample duration is well established as a relevant variable in DMTS research (e.g., Grant, 1976; Roberts \& Grant, 1976), but the influence of sample duration has not been systematically explored in the context of biased-forgetting research. In the case of memory for temporal intervals (e.g., Spetch \& Wilkie, 1983), 
the duration of the sample itself is the relevant dimension. In memory for presence/absence samples, increases or decreases in sample duration might increase or decrease the salience of sample presentations and enhance or retard retention of the memory code. If animals use a single-code strategy, more salient samples should decrease the bias (given a fixed set of test delays), and less salient samples should increase the bias. The relevant experiments have not been carried out.

\section{EXPERIMENT 2}

In a second experiment, pigeons were trained on a new discrimination, in which flicker frequency was explicitly established as the relevant dimension. The birds were taught to make different choices after slow and fast samples followed by psychophysical and retention tests, as in Experiment 1 . In addition, a transfer test was used to determine whether the stimulus coding was accomplished on an absolute or relational basis. The birds might code the absolute values of the stimuli (e.g., $3 \mathrm{~Hz}$ ) and learn to associate each value with the appropriate comparison stimulus. Alternatively, the birds might process the stimuli relationally and associate a relational code (e.g., slow) with the correct comparison. Transfer tests using new stimulus values were used to distinguish between absolute and relational coding (see, e.g., Hulse \& Kline, 1993).

\section{Method}

\section{Subjects}

The subjects were 7 adult male Silver King pigeons maintained at $85 \%$ of their free-feeding weights. Five of the pigeons served in Experiment 1. Two additional pigeons without experience on the regimen of Experiment 1 also served. Both birds had previously served in an experiment involving concurrent fixed-ratio (FR) schedules of reinforcement.

\section{Apparatus}

The apparatus was the same as that in Experiment 1.

\section{Procedure}

At the conclusion of Experiment 1, the 5 subjects were placed on a new task involving a discrimination between different flicker rates, as opposed to the steady versus flickering discrimination in Experiment 1 . Two new pigeons experienced the identical training regimen but were trained and tested after the subjects from Experiment 1 completed the conditions of the second experiment.

Trials began with the illumination of the center key with green light. A peck to the lighted key changed the key color to amber and initiated the sample, which lasted $4 \mathrm{sec}$. On some trials, the sample was a slow flicker rate $(3 \mathrm{~Hz})$, and on other trials, the sample was a fast flicker rate $(9 \mathrm{~Hz})$. The trials were equally divided between slow and fast samples, and the sequencing of sample types was pseudorandom, under the constraint that each block of 12 trials contained equal numbers of each sample. The flickering stimuli consisted of equal light-on and light-off periods. After a sample was presented, the center key was darkened, and the left and right keys were illuminated with red and blue lights; the position of the red and blue lights alternated irregularly across trials. A response to one key color was correct after slow samples, and a response to the al- ternate key color was correct after fast samples; the mapping of sample type (slow vs. fast) to comparison key color was counterbalanced across birds. Correct responses produced $3-\mathrm{sec}$ access to mixed grain, followed by a 10 -second ITI, during which all the lights were off. Incorrect choices produced the ITI directly, and a noncorrection procedure was used. For those birds that had served in Experiment 1, the comparison associated with fast samples was the same as that associated with flickering samples in Experiment 1, and the comparison previously associated with steady samples was now associated with slow samples. Sessions ended after 120 trials.

The acquisition criterion was $80 \%$ correct responses for five consecutive sessions. Five pigeons met the criterion after less than 30 sessions of training, a 6 th required 38 sessions, and 1 pigeon ( $\mathrm{P} 38$ ) required 73 sessions to achieve the criterion. Ten additional sessions of training were given after the criterion was met.

Psychophysical probe test. Once the basic discrimination was established, the task was modified so that $50 \%$, rather than $100 \%$, of correct choices yielded reinforcement. Correct choices on nonreinforced trials simply produced the ITI, as did all incorrect choices. Probe trials were instituted after five sessions of training under the partial reinforcement schedule. Probe trials consisted of flicker rates intermediate to the training values, and the probe trials made up $50 \%$ of the trials in each test session. Four probe stimuli were used $(3.5,4,5$, and $6 \mathrm{~Hz})$; each probe stimulus occurred 15 times during each test session. The training and test values were well below the pigeon's fusion threshold (Powell \& Smith, 1968). Choices on probe trials were recorded but never reinforced. Probe sessions alternated with training sessions, providing 50\% reinforcement for choices, and a total of five probe sessions were conducted.

Delay test. Once psychophysical testing was completed, all the pigeons were returned to the original testing conditions that provided two sample types, slow $(3 \mathrm{~Hz})$ and fast $(9 \mathrm{~Hz})$ and reinforcement for each correct choice. After five sessions of reminder training, delay testing was initiated. During delay test sessions, $50 \%$ of the trials included a delay between the termination of the sample and the presentation of the comparison stimuli; all the lights were off during delay intervals. Delays of 2, 5, and $12 \mathrm{sec}$ were used, and each delay and sample type occurred equally often within each session (i.e., 10 presentations of each sample type at each delay). The remaining trials involved an immediate $(0-\mathrm{sec})$ test of sample discrimination. Immediate and delayed test trials were randomly intermixed within sessions, and delay testing lasted five sessions.

Transfer. When delay testing was completed, the birds were returned to the original training conditions for five sessions $(0-\mathrm{sec}$ delay on all trials). Then, the values of the stimuli were changed, and the birds acquired a discrimination between a different pair of flicker values. Under the new discrimination, the slow sample was $9 \mathrm{~Hz}$, and the fast sample was $27 \mathrm{~Hz}$, maintaining the same ratio of flicker rates (3:1) but changing the absolute values of the samples. Note that the value of the new slow sample $(9 \mathrm{~Hz})$ equalled the value of the fast sample in the previous condition. For 3 pigeons (P18, P47, and P91), the choices associated with fast and slow samples were reversed from the previous condition; the effect of the reversal was to maintain a constant sample-choice association for the 9-Hz sample (absolute transfer). If sample-choice associations are based on absolute flicker rates, the choice reversal should yield positive transfer, as compared with a condition in which relative sample-choice associations were maintained. For the other 3 birds (P12, P49, and P54; P38 became ill and was dropped from the experiment), choices associated with fast and slow samples were the same as those for the previous condition, maintaining a constant association between sample type (slow vs. fast) and choice (relational transfer).

A .05 level of significance was used for all statistical comparisons. All post hoc comparisons were carried out according to Tukey's procedures for comparisons of unconfounded means. 


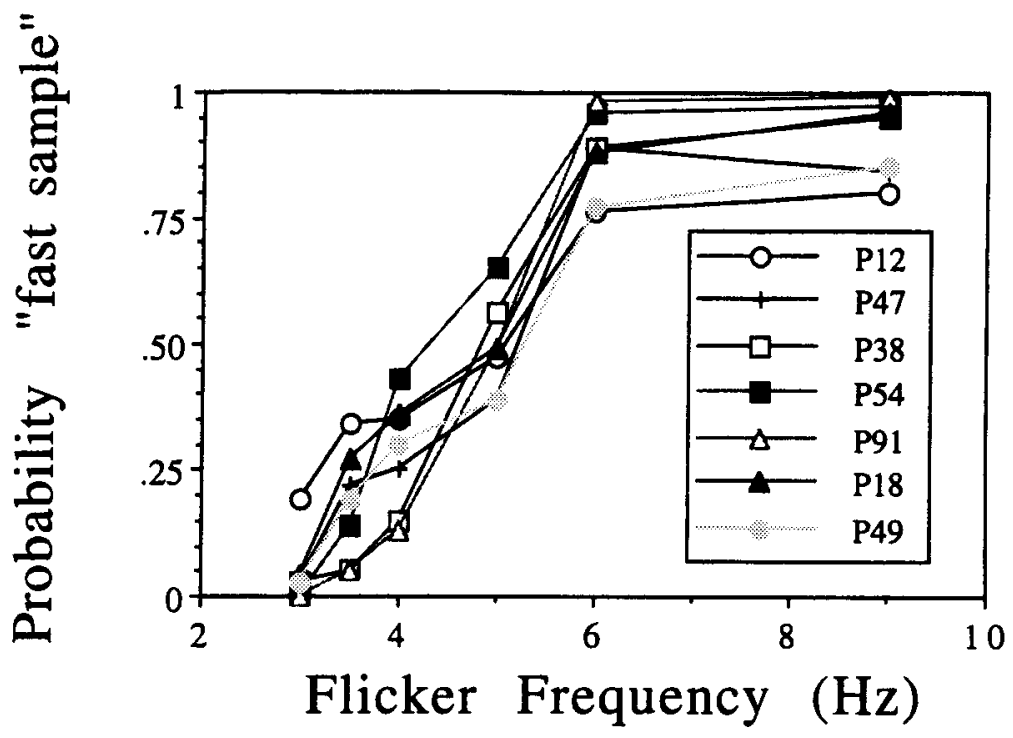

Figure 5. Probability of responding to the alternative associated with the fast sample as a function of the flicker frequency of the training and test stimuli. Probabilities were calculated by dividing the number of choices of the alternative with the fast sample at each stimulus value by the total number of occurrences of each value. Data are shown for individual pigeons, represented by different symbols.

\section{Results}

Figure 5 shows the the psychophysical functions for the flicker frequency discrimination. All the birds displayed a characteristic graded ogival pattern relating choices to stimulus value. The slopes for 2 subjects (P12 and P47) were less steep than those for the other pigeons, but all the birds showed good control by the sample dimension. In the temporal domain, bisection points for psychometric functions typically fell near the geometric mean of the training stimuli (see, e.g., Allan \& Gibbon, 1991; Church \& Deluty, 1977; Fetterman \& Killeen, 1992). Flicker frequency is a higher order dimension consisting of temporal components (equal light-on and light-off periods), and thus, it might not be too surprising if the birds bisected at the geometric mean of the training values. Bisection points were estimated as a parameter of a cumulative normal distribution (CND) fitted to the psychometric function for each pigeon. The fitted CNDs accounted for a substantial proportion of the data variance (mean $=.96$; range, $.88-.99$ ). The recovered bisection points were very similar for the 7 birds, falling between the harmonic (4.5) and the geometric (5.20) means of the stimuli (mean $=4.89$; range, 4.46-4.96).

Figure 6 shows the retention data separately for slow $(3 \mathrm{~Hz})$ and fast $(9 \mathrm{~Hz})$ samples. As in Experiment 1 , there was an asymmetry in forgetting. Five of 7 pigeons showed better retention of slow than of fast samples. The data for Pigeons 38, 54, 91, 18, and 49 provided a clear demonstration of this effect (although the pattern for P38 was slightly muddled by a strong sample bias at the 0 -sec delay); the effect for P12 was less strong, and that for
P47 was nonexistent. Inferential statistics are useful in distinguishing signal from noise in instances like these. A two-way ANOVA revealed significant main effects of sample type $[F(1,6)=7.78]$ and delay $[F(3,18)=17.58]$ and a significant interaction $[F(3,18)=5.59]$, the signature of biased forgetting. A post hoc test showed that the sample type accuracy difference was significant at all delays, except the 0 -sec delay. ${ }^{1}$

Because the performance of $\mathrm{P} 47$ was insensitive to the range of test delays displayed in Figure $6(0,2,5$, and $12 \mathrm{sec}$ ), this pigeon was given a second round of delay testing (for five sessions) with longer delays $(0,4,10$, and $20 \mathrm{sec}$ ). Figure 7 shows that P47 did display a bias for the slow sample when tested with the extended range of delays.

Figure 8 shows the outcome of transfer to the new $(9$ vs. $27 \mathrm{~Hz}$ ) flicker rate discrimination. The figure shows accuracy for the last 3 sessions under the original discrimination and for the first 12 sessions of the new discrimination. Pigeons receiving a choice reversal (P18, $\mathrm{P} 47$, and P91, absolute transfer) showed positive transfer to the new discrimination (and P47 and P91 showed essentially perfect transfer). When the relation between sample type and choice was maintained (P12, P49, and P54, relational transfer) birds exhibited negative transfer to the new discrimination.

\section{Discussion}

The pigeons showed a statistically reliable tendency to select the comparison stimulus associated with the slow (3-Hz) sample (a choose-slow effect), a tendency that increased with lengthening delays. This result seemingly 

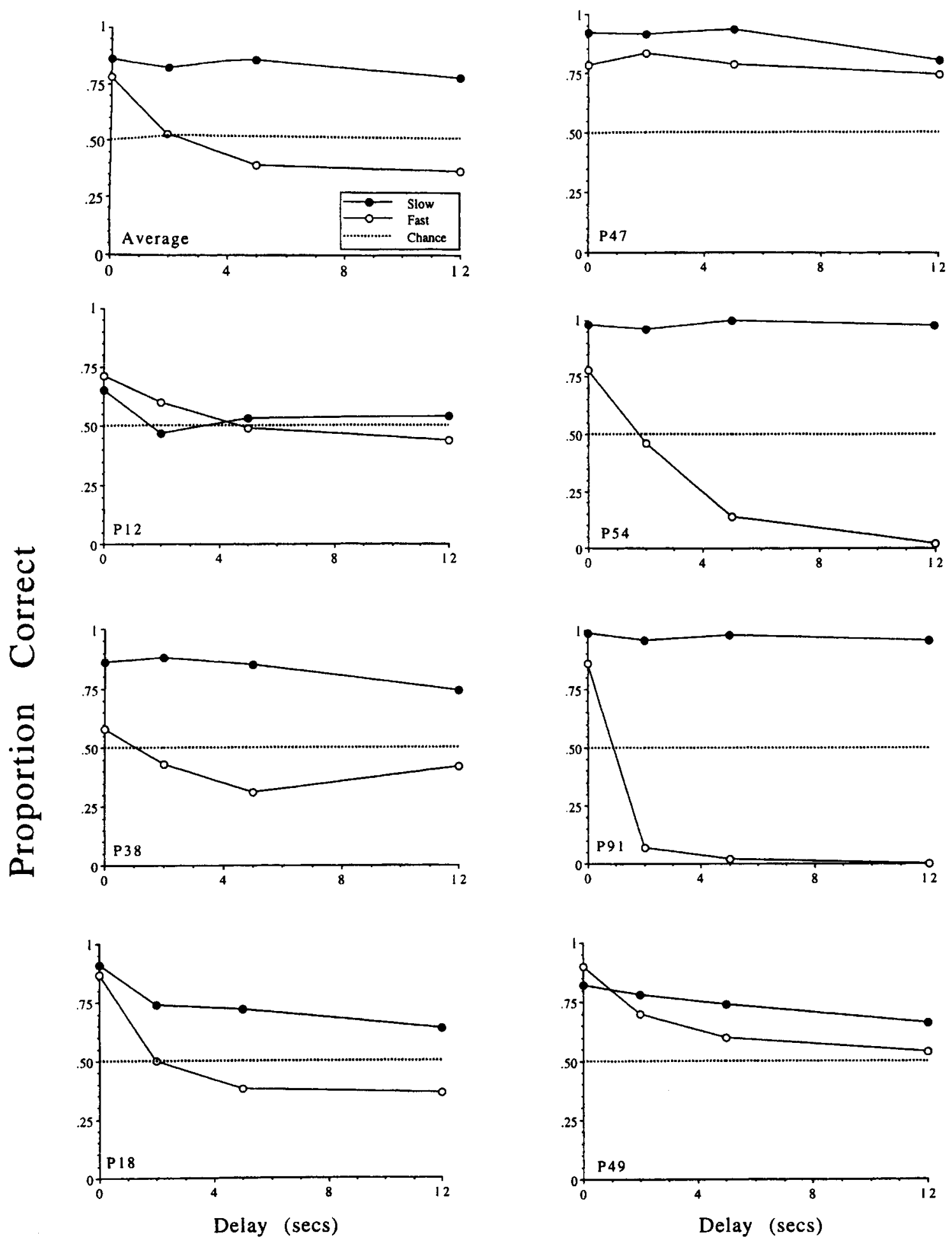

Figure 6. Proportion of correct choices as a function of the delay between the sample stimulus and the comparison stimuli. The data are shown separately for trials initiated by a fast sample and those initiated by a slow sample.

contradicts the findings of Honig and Spetch (1988), who found that pigeons tended to select the comparison associated with a fast sample. But there are some important procedural differences between the two experiments, and the differences may have resulted in different memory strategies. First, Honig and Spetch used relatively slow rates of stimulus change (one cycle per second against one cycle every $4 \mathrm{sec}$ ), whereas the rates of change were faster in the present experiment ( 3 and $9 \mathrm{~Hz}$ ). Second, Honig and Spetch used red and green 


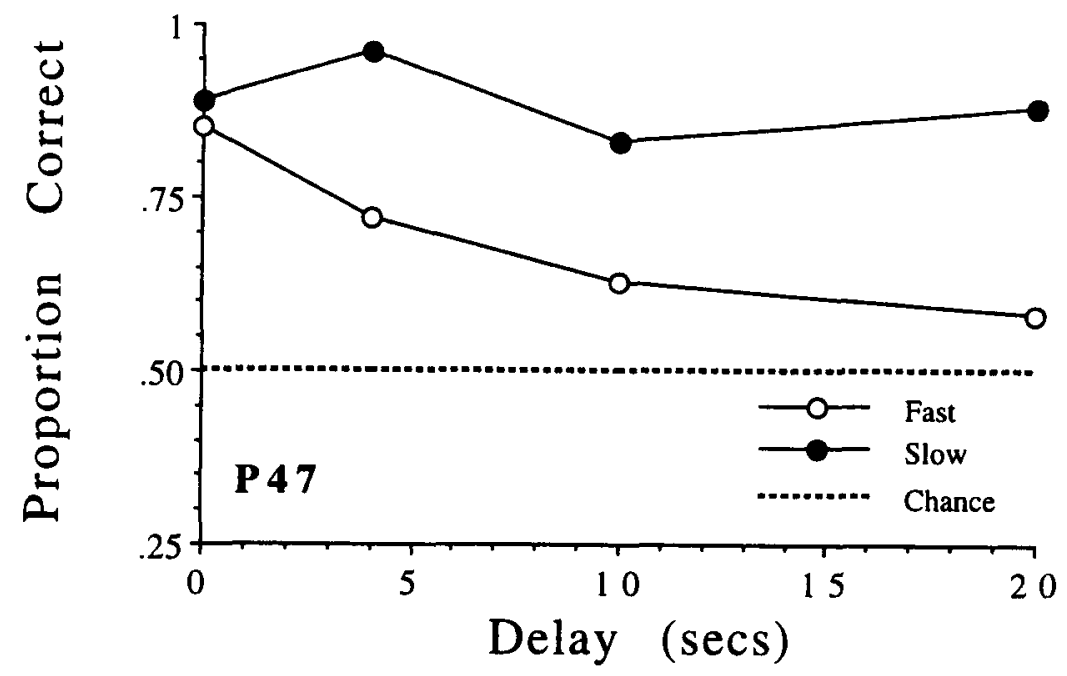

Figure 7. Proportion of correct choices as a function of an extended series of delays $(0,4,10$, and 20 sec) between the sample stimulus and the comparison stimuli for $P 47$. The data are shown separately for trials initiated by a fast sample and those initiated by a slow sample.

hues to identify the components of each cycle, whereas the cycles in the present experiment consisted of equal light-on and light-off periods. Honig and Spetch interpreted their findings as reflecting a subjective shortening of the constituent cycle durations, but the present method of identifying cycles by stimulus onset and offset may have induced a different coding strategy.

Suppose the pigeon registered each presentation of the light as an event and accumulated a tally of events over the duration of each 4-sec sample. Under such circumstances, the comparison choices would be associated with different numbers of events, and the task would entail a discrimination of numerosities, or few versus many. Under this interpretation, the bias for the slow comparison is, rather, a bias for the few (or small) comparison. There is some precedent in the literature for this interpretation.

Fetterman and MacEwen (1989) found that pigeons tended to select a comparison associated with a "sample" consisting of a small FR requirement more frequently than one associated with a larger FR when the comparisons were delayed from the samples. But, in their experiment, the FR sample requirement was confounded with the time taken to complete the requirement. Thus, the birds could have timed, rather than counted, the samples. Roberts et al. (1995) trained pigeons on a numerosity discrimination in which the durations of the different numerosities were equated. In their experiment, pigeons experienced a sequence of light flashes during a 4-sec sample period. On some trials, the sample period contained two light flashes, and on others, the sample contained eight flashes (note that the rate of flashes differed for the two samples, as did the interflash interval). When the comparison stimuli were delayed from the light-flash samples, the birds tended to report that the prior sample contained two (fewer) as opposed to eight flashes, very much like the present results.
Roberts et al. (1995; see also Roberts, 1997) offered an extension of a mode-control model of timing and counting first proposed by Meck and Church (1983; see Meck, 1997, for an update) to account for their results. The essence of the model is that both time and number are represented in working memory as a tally of counts or pulses and that counts are lost from memory during delays between a sample and a presentation of the choices. Thus, over a lengthy delay, the working memory for a large sample increasingly comes to resemble the stored reference memory value of a small sample, resulting in the bias to report that the smaller (or shorter) of two samples was just presented.

The transfer test to new (9 vs. $27 \mathrm{~Hz}$ ) frequency values provided unambiguous evidence that the pigeons based choices on the absolute values of the stimuli (Figure 8). All 3 birds that received the absolute transfer regimen showed positive transfer. Moreover, for 2 of these birds, accuracy levels on the 1 st day of transfer remained about the same as that under the prior discrimination. By contrast, accuracy decreased to chance level or below for birds given relational transfer.

Hulse and Kline (1993) studied a very similar discrimination and obtained the opposite result. In their task, starlings were trained to discriminate between different rates of sound pulses. The birds were trained on a baseline discrimination between pulse trains delivered at the rate of $4 / \mathrm{sec}$ (slow) and $8 / \mathrm{sec}$ (fast). Once the discrimination was acquired, the starlings were transferred to a new discrimination between pulse trains of $8 / \mathrm{sec}$ and $16 / \mathrm{sec}$. Some birds were given absolute transfer (choice associated with the $8 / \mathrm{sec}$ pulse trained remained constant), and others were given relational transfer (choices associated with slow and fast tempos remained constant). Starlings that underwent relational transfer showed significant positive transfer, whereas those that under- 

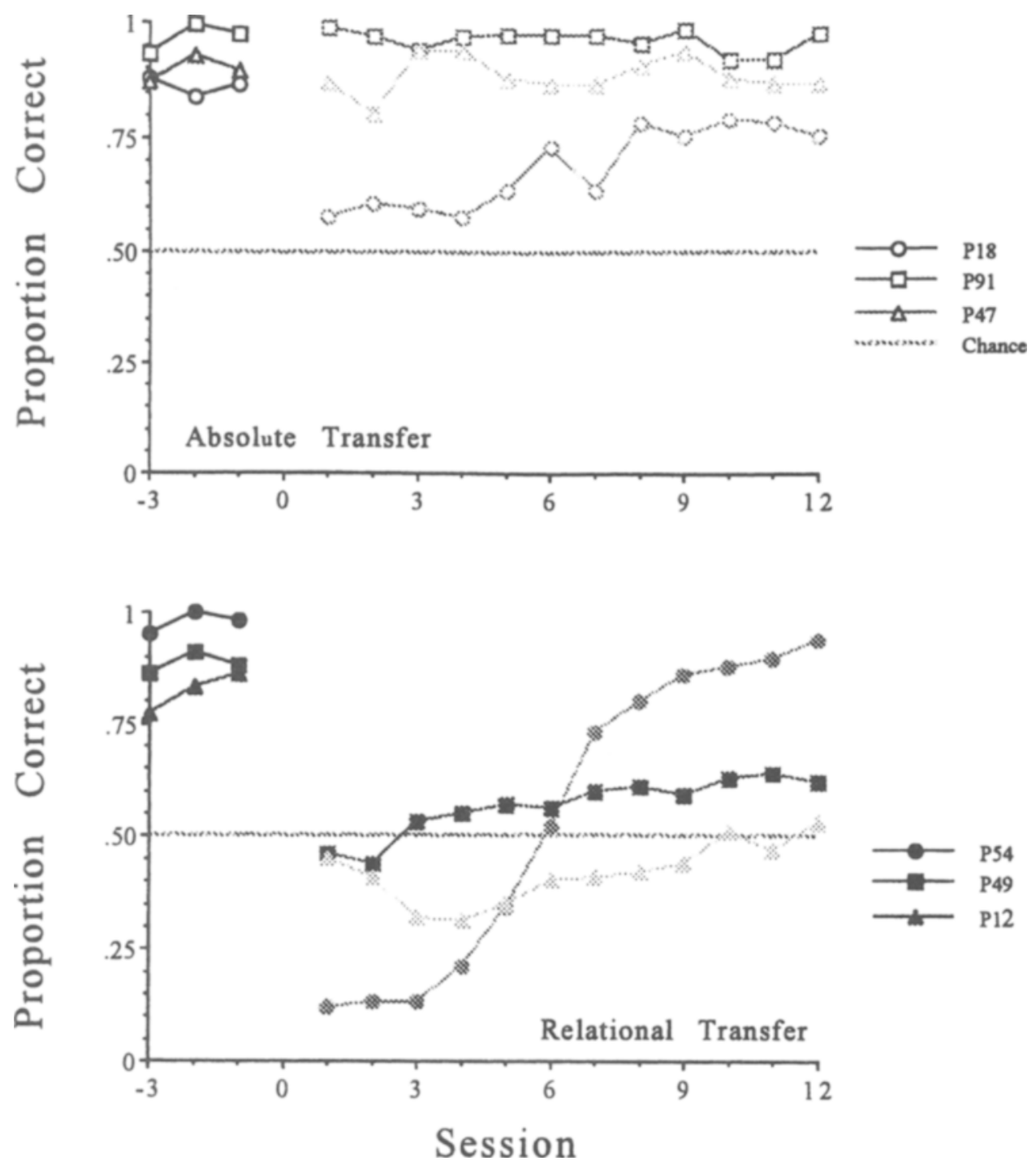

Figure 8. Accuracy (proportion of correct choices) before and after transfer from one frequency discrimination $(3 \mathrm{vs.} 9 \mathrm{~Hz}$ ) to another $(9 \mathrm{vs.} 27 \mathrm{~Hz})$. The pigeons in the bottom panel were given relational transfer, and those in the top panel were given absolute transfer (see the text for details).

went absolute transfer showed significant negative transfer. It is difficult to know what to make of the differences between the present results and those of Hulse and Kline. They may reflect dimensional differences in stimulus processing (sounds vs. lights), species differences (starlings vs. pigeons), or more likely, an interaction between these two variables.

\section{GENERAL DISCUSSION}

Two experiments using a DMTS task demonstrated differential rates of forgetting according to sample type. In Experiment 1, pigeons discriminated between steady and flickering lights and showed better retention of the flickering sample, a pattern that was not altered by changes in sample duration. In Experiment 2, the birds discrimi- nated between different rates of stimulus change, showing better retention for the slow sample. These findings add to a literature on biased forgetting that includes delayed discriminations of stimulus duration (e.g., Fetterman, 1995; Spetch \& Wilkie, 1983), numerosities (e.g. Fetterman \& MacEwen, 1989; Roberts et al., 1995), and presence/absence samples (e.g., Grant, 1991; Sherburne \& Zentall, 1993).

A theory of discriminating and remembering based on event salience (Wixted \& Dougherty, 1996) can account for some, but not all of the results. If, as is hypothesized, the discrimination in Experiment 2 was based on the number of sample events (light flashes), the observed bias represents an instance of choose-small effects, reported by Fetterman and MacEwen (1989) and Roberts et al. (1995). These effects are consistent with Wixted and 
Dougherty's account, on the plausible assumption that the sample containing the larger number of events is more salient than the sample containing the smaller number.

It is less obvious, however, why pigeons should remember a flickering light better than a steady light (Experiment 1). On the face of it, a flickering light seems more salient than a steady light and, thus, more susceptible to the deleterious effects of memory delays. Nonetheless, all the pigeons remembered flickering samples better than steady samples. The crux of the matter is that methods for evaluating event salience must be specified and implemented in advance of retention tests. It may be that a steady light is more salient for the pigeon than is a flickering light, but the only evidence for this counterintuitive assertion is the observed retention pattern. Some manipulations may afford straightforward tests of the model, as, for instance, in situations involving differences in the intensive properties of stimuli. It is relatively simple to train animals to discriminate low- from highintensity values (e.g., soft vs. loud sounds or dim vs. bright lights). If it is assumed that salience is related to stimulus intensity, delayed tests of intensity discriminations should reveal biases for the comparison associated with the less intense (salient) sample.

The present results add to a now substantial literature on biases in pigeon working memory, but they do not identify a single mechanism responsible for all instances of memory biases, at least not at the present time. Whereas a theory based on event salience may explain some aspects of the data, it cannot, in its current form, accommodate the basic result of Experiment 1. Whatever the mechanism(s), investigations of biased forgetting have provided fertile ground for exploring working memory processes in pigeons and other animals.

\section{REFERENCES}

Allan, L. G., \& GibBon, J. (1991). Human bisection at the geometric mean. Learning \& Motivation, 22, 39-58.

BLough, D. S. (1959). Delayed matching in the pigeon. Journal of the Experimental Analysis of Behavior, 2, 151-160.

Church, R. M., \& Deluty, M. Z. (1977). Bisection of temporal intervals. Journal of Experimental Psychology: Animal Behavior Processes, 3, 216-227.

Fetterman, J. G. (1995). The psychophysics of remembered duration. Animal Learning \& Behavior, 23, 49-62.

Fetterman, J. G., \& Killeen, P. R. (1992). Time discrimination in Columba livia and Homo sapiens. Journal of Experimental Psychology: Animal Behavior Processes, 18, 80-94.

FetTerman, J. G., \& MaCEwEn, D. (1989). Short-term memory for responses: The "choose-small" effect. Journal of the Experimental Analysis of Behavior, 52, 311-324.

GraNT, D. S. (1976). Effect of sample presentation time on long-delay matching in the pigeon. Learning \& Motivation, 7, 580-590.

Grant, D. S. (1991). Symmetrical and asymmetrical coding of food and no-food samples in delayed matching in pigeons. Journal of $E x$ perimental Psychology: Animal Behavior Processes, 17, 186-193.

Grant, D. S., SPETCh, M. L., \& Kelly, R. (1997). Pigeons' coding of event duration in delayed-matching-to-sample. In C. M. Bradshaw \&
E. Szabadi (Eds.), Time and behaviour: Psychological and neurobehavioural analyses. Amsterdam: Elsevier.

HoNiG, W. K., \& SPETCH, M. (1988). Short-term memory for rate of alternation in the pigeon. Bulletin of the Psychonomic Society, 26, 152154.

Hulse, S. H., \& KLINE, C. L. (1993). The perception of time relations in auditory tempo discrimination. Animal Learning \& Behavior, 21, 281-288.

Kendrick, D. F., Rilling, M., \& DenNy, R. (ED.) (1986). Theories of animal memory. Hillsdale, NJ: Erlbaum.

Macmillan, N. A., \& Creelman, C. D. (1991). Detection theory: A user's guide. Cambridge: Cambridge University Press.

Meck, W. H. (1997). Application of a mode-control model of temporal integration to counting and timing behavior. In C. M. Bradshaw \& E. Szabadi (Eds.), Time and behaviour: Psychological and neurobehavioural analyses. Amsterdam: Elsevier.

Meck, W. H., \& Church, R. M. (1983). A mode control model of counting and timing processes. Journal of Experimental Psychology: Animal Behavior Processes, 9, 320-334.

Powell, R. W., \& SMiтh, J. C. (1968). Critical flicker fusion thresholds as a function of very small pulse to cycle fractions. Psychological Record, 18, 35-40.

ROBERTS, W. A. (1997). Does a common mechanism account for timing and counting phenomena in the pigeon? In C. M. Bradshaw \& E. Szabadi (Eds.), Time and behaviour: Psychological and neurobehavioural analyses. Amsterdam: Elsevier.

RoberTs, W. A., \& Grant, D. S. (1976). Studies of short-term memory in the pigeon using the delayed matching-to-sample procedure. In D. L. Medin, W. A. Roberts, \& R. T. Davis (Eds.), Processes of animal memory (pp. 79-112). Hillsdale, NJ: Erlbaum.

Roberts, W. A., Macuda, T., \& Brodbeck, D. R. (1995). Memory for number of light flashes in pigeons. Animal Learning \& Behavior, 23, 182-188.

Sherburne, L. M., \& Zentall, T. R. (1993). Asymmetrical coding of food and no-food events by pigeons: Sample pecking versus food as the basis of the sample code. Learning \& Motivation, 24, 141-155.

SPeTCH, M. L., \& Rusak, B. (1992). Time present and time past. In W. K. Honig \& J. G. Fetterman (Eds.), Cognitive aspects of stimulus control (pp. 47-67). Hillsdale, NJ: Erlbaum.

SPetCh, M. L., \& Wilkie, D. M. (1983). Subjective shortening: A model of pigeons' memory for event duration. Journal of Experimental Psychology: Animal Behavior Processes, 9, 14-30.

Urculoli, P. J., \& Zentall, T. R. (1986). Retrospective coding in pigeons' delayed matching-to-sample. Journal of Experimental Psychology: Animal Behavior Processes, 12, 69-77.

WiXTED, J. T. (1993). A signal detection analysis of memory for nonoccurrence in pigeons. Journal of Experimental Psychology: Animal Behavior Processes, 19, 400-411.

WiXTED, J. T., \& DovgherTy, D. H. (1996). Memory for asymmetric events. In D. L. Medin (Ed.), The psychology of learning and motivation (Vol. 35, pp. 89-126). San Diego: Academic Press.

ZENTALL, T. R. (1997). Animal memory: The role of "instructions." Learning \& Motivation, 28, 248-267.

\section{NOTE}

1. Note that most of the pigeons (P12 and P49 are the exceptions) were more accurate after slow than after fast samples at the 0-sec test delay (mean difference equals .09). The difference was not statistically reliable, as is indicated by the post hoc test. However, there was a significant change in bias on $0-\mathrm{sec}$ delay trials before and after the introduction of the retention intervals. The mean difference in accuracy was .017 for the five sessions immediately preceding delay testing and .016 for the five sessions after delay testing was completed.

(Manuscript received January 27, 1999 revision accepted for publication August 23, 1999.) 